\title{
Direct Data Domain Sparsity-Based STAP Utilizing Subaperture Smoothing Techniques
}

\author{
Zhaocheng Yang, ${ }^{1}$ Rui Fa, ${ }^{2}$ Yuliang Qin, ${ }^{1}$ Xiang $\mathrm{Li},{ }^{1}$ and Hongqiang Wang ${ }^{1}$ \\ ${ }^{1}$ Research Institute of Space Electronics, Electronics Science and Engineering School, \\ National University of Defense Technology, Changsha 410073, China \\ ${ }^{2}$ Department of Electrical Engineering and Electronics, The University of Liverpool, Liverpool L69 3GJ, UK
}

Correspondence should be addressed to Zhaocheng Yang; yangzhaocheng@gmail.com

Received 25 April 2014; Revised 6 October 2014; Accepted 16 October 2014

Academic Editor: Hang Hu

Copyright (C) 2015 Zhaocheng Yang et al. This is an open access article distributed under the Creative Commons Attribution License, which permits unrestricted use, distribution, and reproduction in any medium, provided the original work is properly cited.

\begin{abstract}
We propose a novel direct data domain (D3) sparsity-based space-time adaptive processing (STAP) algorithm utilizing subaperture smoothing techniques for airborne radar applications. Different from either normal sparsity-based STAP or D3 sparsity-based STAP, the proposed algorithm firstly uses only the snapshot in the cell under test (CUT) to generate multiple subsnapshots by exploiting the space-time structure of the steering vector and the uncorrelated nature of the components of the interference covariance matrix. Since the interference spectrum is sparse in the whole angle-Doppler plane, by employing a sparse regularization, the generated multiple subsnapshots are jointly used to recover the interference spectrum. The interference covariance matrix is then estimated from the interference spectrum, followed by the space-time filtering and the target detection. Simulation results illustrate that the proposed algorithm outperforms the generalized forward/backward method, the conventional D3 least squares STAP algorithm, and the existing D3 sparsity-based STAP algorithm. Furthermore, compared with the normal sparsity-based STAP algorithm using multiple snapshots, the proposed algorithm can also avoid the performance degradation caused by discrete interferers merely appearing in the CUT.
\end{abstract}

\section{Introduction}

Space-time adaptive processing (STAP) is considered to be an effective tool for detection of weak targets by airborne radar systems in strong interference environments [1-4]. The problem is essentially that it usually assumes a homogeneous environment over the range cells. However, the assumptions are often not satisfied in realistic radar scenarios. Many factors, such as clutter edges, moving scatterers, shadowing and obstruction, and chaff, can render the clutter returns nonhomogeneous, resulting in performance degradation of conventional STAP algorithms [4].

Direct data domain least-squares (D3-LS) STAP approach is considered to be a powerful and effective method in nonhomogeneous environments [5-8]. It only uses the snapshot in the cell under test (CUT) rather than training data, which can avoid the nonhomogeneity in the training data and eliminate the impacts of nonhomogeneous environments. However, this configuration degrades the system performance since the system degrees of freedom (DOFs) are reduced. By combining the STAP algorithms using the training data and the D3-LS STAP algorithms, the hybrid detection approach is introduced with improved robustness to nonhomogeneous environments $[9,10]$. Knowledge-aided (KA) STAP approaches using digital land classification data and digital elevation data were proposed to select training data to obtain improved STAP performance [11]. Another KA-STAP method, called model-based approach (see [12-19] and the references therein), basically employs some prior knowledge to form the simplified general clutter model (GCM) and then blends the GCM with the measured observations to design the STAP filter or directly uses it to design the STAP filter. This method can obtain good performance in a small-samplesupport condition with accurate prior knowledge. However, the formed GCM usually does not contain the information of discrete interferers because the discrete interferers can appear at arbitrary positions in the angle-Doppler plane, which results in the increase of false alarms. Moreover, in practice, 
one cannot guarantee the accuracy of the prior knowledge, which is very important to the performance.

Recently, sparse recovery (SR) methods have been considered for STAP problems, such as sparsity-based STAP algorithms in [20-29] and L1-regularized STAP filters in [30, 31]. The sparsity-based STAP algorithms are highly related to the model-based approach but do not require the knowledge (such as the clutter ridge) to form the GCM and can be applied to arbitrary array geometries and random slow-time samples [21]. In fact, supposing that no prior knowledge of the interference is available, they discretize the whole angleDoppler plane into many small grids, exploit the sparsity of the interference spectrum in all discretized angle-Doppler grids, and utilize the sparse recovery algorithms to recover the interference spectrum. Usually, there are two types of sparsity-based STAP in the literature: one type is the D3 STAP based on sparse recovery (D3-SR-STAP) algorithm which uses only the snapshot in the CUT [20-24]; the other is the normal STAP based on sparse recovery (NSR-STAP) that uses multiple snapshots adjacent to the CUT [24-29]. Both types exhibit significantly better performance than conventional STAP algorithms in limited training situations. However, for the D3-SR-STAP, the estimated interference covariance matrix is not stable since only one snapshot is employed; for the NSR-STAP, the presence of discrete interferers merely in the CUT will degrade the performance significantly.

In this paper, we propose a new D3-SR-STAP using the subaperture smoothing (SASM) techniques, which can overcome aforementioned drawbacks of both types of sparsitybased STAP. The proposed algorithm uses only snapshot in the CUT as conventional D3-SR-STAP does. Additionally, it uses the decimation techniques $[32,33]$ to generate multiple subsnapshots by exploiting the space-time structure of steering vector and the uncorrelated nature of the components of the interference covariance matrix. Then, the generated multiple subsnapshots are jointly used to recover the interference spectrum. Inspired by the SASM techniques used in the spectral estimation [34], the proposed algorithm can reduce the variance of the estimated interference spectrum resulting in improved signal-to-interference-plus-noise ratio (SINR). Moreover, thanks to the rapid convergence of sparsity-based STAP algorithms $[25,26,28]$, the proposed algorithm does not reduce much the number of DOFs to increase the subsnapshots, which will benefit the recovery of the interference spectrum from single subsnapshot. It should be noted that another method, called the generalized forward/backward (F/B) method [35], also employs the SASM techniques and uses multiple training snapshots adjacent to the CUT to generate multiplicative improvement in the snapshots resulting in improved performance in sample limited cases. However, when the training snapshots have different statistics with that in the CUT, the performance of the generalized F/B method will degrade significantly. Simulation results illustrate the effectiveness of the proposed algorithm.

The rest of the paper is organized as follows. In Section 2, the STAP signal model and the principle of sparsity-based STAP algorithms are introduced. Section 3 details the proposed SASM D3-SR-STAP algorithm. Simulated data are used to evaluate the performance of the proposed algorithm in Section 4. Section 5 provides the summary and conclusions.

\section{Signal Model and Problem Formulation}

In this section, we will introduce the signal model used in the paper and discuss the principle of the sparsity-based STAP algorithms.

2.1. Signal Model. In airborne radar systems, ignoring the impact of range ambiguities, a general model for the spacetime interference (clutter and discrete interferers) plus noise snapshot $\mathbf{x}$ in a target-free range cell is given by [3]

$$
\begin{aligned}
\mathbf{x}= & \mathbf{x}_{c}+\mathbf{x}_{I}+\mathbf{n}=\sum_{n=1}^{N_{c}} \sigma_{c ; n} \mathbf{v}\left(f_{c ; d, n}, \phi_{c ; n}\right) \\
& +\sum_{m=1}^{N_{I}} \sigma_{I ; m} \mathbf{v}\left(f_{I ; d, m}, \phi_{I ; m}\right)+\mathbf{n},
\end{aligned}
$$

where $\mathbf{n}$ is the Gaussian white noise vector with the noise power $\sigma_{n}^{2}$ on each channel and pulse; $N_{c}$ and $N_{I}$ denote the numbers of independent clutter patches and independent discrete interferers over the iso-range of interest; $\sigma_{c ; n}, \phi_{c ; n}$, and $f_{c ; d, n}$ are the random complex amplitude, the angle-ofarrival (AOA), and the Doppler frequency of the $n$th clutter patch, respectively; $\sigma_{I ; m}, \phi_{I ; m}$, and $f_{I ; d, m}$ are the random complex amplitude, the AOA, and the Doppler frequency of the $m$ th discrete interferer, respectively; $\mathbf{v}\left(f_{d}, \phi\right)$ is the $N M \times 1$ space-time steering vector with the AOA $\phi$ and the Doppler frequency $f_{d}$ and is defined by (for a uniform linear array (ULA))

$$
\mathbf{v}\left(f_{d}, \phi\right)=\left[\begin{array}{c}
1 \\
\vdots \\
e^{j 2 \pi(M-1) f_{s}} \\
e^{j 2 \pi f_{d}} \\
\vdots \\
e^{j 2 \pi\left((N-1) f_{d}+(M-1) f_{s}\right)}
\end{array}\right],
$$

where $N$ is the number of pulses in a coherent process interval (CPI), $M$ is the number of array elements, and $f_{s}$ corresponds to the spatial frequency related to the AOA $\phi$. Let the inner spacing of array elements be $d_{a}$; then the relationship between the spatial frequency and the AOA is $f_{s}=\left(d_{a} / \lambda_{c}\right) \sin \phi$, where $\lambda_{c}$ is the operating wavelength.

2.2. Principle of Sparsity-Based STAP Algorithms. Recently developed sparsity-based STAP algorithms provide an effective approach to estimate interference (clutter, discrete interferers) covariance matrix. It first discretizes the whole angleDoppler plane into $N_{s}=\rho_{s} M, N_{d}=\rho_{d} N\left(\rho_{s}, \rho_{d}>1\right)$ grids, where $N_{s}$ and $N_{d}$ are the number of angle and Doppler bins, respectively. Then the received data in (1) can be rewritten as [22-29]

$$
\mathbf{x}=\boldsymbol{\Phi} \gamma+\mathbf{n}
$$


where $\boldsymbol{\gamma}=\left[\gamma_{1,1}, \gamma_{1,2}, \ldots, \gamma_{N_{d}, N_{s}}\right]^{T}$ denotes the $N_{d} N_{s} \times 1$ angle-Doppler profile with nonzero elements representing the interference, and the $N M \times N_{d} N_{s}$ matrix $\Phi$ is the overcompleted space-time steering dictionary including all the possible space-time steering vectors, as given by

$$
\Phi=\left[\mathbf{v}\left(f_{d, 1}, \phi_{1}\right), \ldots, \mathbf{v}\left(f_{d, 1}, \phi_{N_{s}}\right), \ldots, \mathbf{v}\left(f_{d, N_{d}}, \phi_{N_{s}}\right)\right] .
$$

Here, the symbols $f_{d, i}, 1 \leq i \leq N_{d}$ and $\phi_{k}, 1 \leq k \leq N_{s}$ denote the uniformly quantized Doppler frequencies and the AOAs. Herein, the interference plus noise covariance matrix can be expressed as

$$
\mathbf{R}=E\left[\mathbf{x x}^{H}\right]=\boldsymbol{\Phi} \Gamma \boldsymbol{\Phi}^{H}+\sigma_{n}^{2} \mathbf{I},
$$

where $\boldsymbol{\Gamma}=E\left[\gamma \gamma^{H}\right]=\operatorname{diag}(\mathbf{a})$ and $\mathbf{a}=E\left[\gamma \odot \gamma^{*}\right]$ is the interference spectral distribution vector under the assumptions of statistical independence between the clutter patches, the discrete interferers, and the noise $[3,4]$. Here, $\operatorname{diag}(\mathbf{a})$ stands for a diagonal matrix with the main diagonal taken from the elements of the vector $\mathbf{a}$, and $\odot$ denotes the Hadamard product.

To estimate $\mathbf{R}$, the sparsity-based STAP algorithms try to calculate the parameter a using the computed angle-Doppler profile from the CUT or angle-Doppler profiles from adjacent target-free range cells, which can be obtained via the sparse recovery algorithms by exploiting the intrinsic sparsity of the interference spectral distribution, for example, the sparse interference spectrum computed by using the Mountain Top dataset [36], as shown in Figure 1. The whole angle-Doppler plane shows a high degree of sparsity and only a small number of angle-Doppler grids of the interference spectrum are occupied. Mathematically, the angle-Doppler profile can be estimated by solving the following minimal optimization problem known as the least absolute shrinkage and selection operator (LASSO) [23-26]

$$
\widehat{\gamma}=\arg \min _{\gamma}\|\gamma\|_{1} \quad \text { s.t. }\|\mathbf{x}-\Phi \boldsymbol{\gamma}\|_{2}^{2} \leq \epsilon,
$$

or the basis pursuit denoising (BPDN) [20, 22, 24, 26, 27, 29], given as

$$
\widehat{\gamma}=\arg \min _{\gamma}\left\{\frac{1}{2}\|\mathbf{x}-\boldsymbol{\Phi} \boldsymbol{\gamma}\|_{2}^{2}+\kappa\|\gamma\|_{1}\right\},
$$

where $\|\cdot\|_{p}(p=1,2)$ denotes the $l_{p}$-norm, $\epsilon$ is the noise error allowance, and $\kappa$ is a positive regularization parameter that provides a tradeoff between the approximation error and the sparsity.

For the D3-SR-STAP algorithm in [20-24], it is hard to obtain a stable and good estimation of interference covariance matrix estimate by using only one snapshot. For the NSRSTAP algorithm using multiple snapshots [24-29], although it can obtain a good performance by effectively suppressing the interference in homogeneous environments, it will lead to significant performance degradation in presence of discrete interferers only in the CUT. It is worthy of noting that, for the developed algorithm in [22], the exact prior knowledge

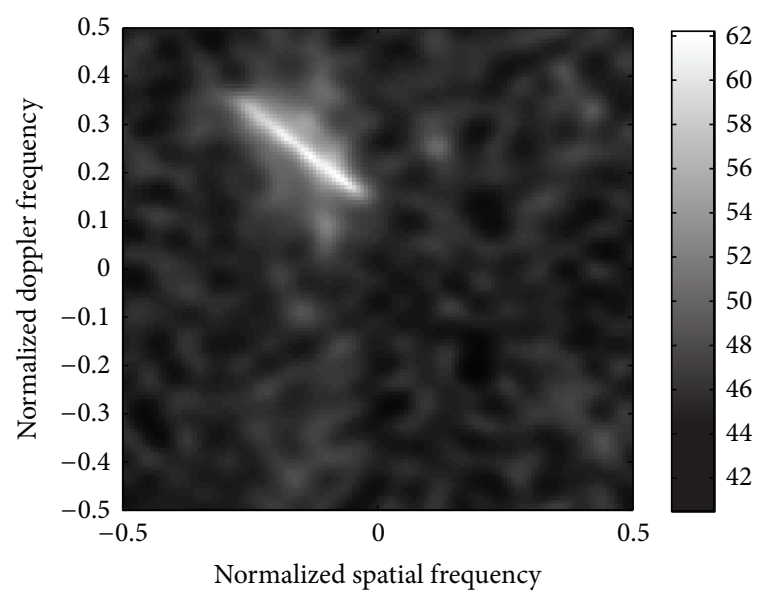

FIgURE 1: The estimated interference spectrum using the Mountain Top dataset.

of the clutter ridge in the angle-Doppler plane is required; however, it is hard to obtain in practice. Thus, we focus on sparsity-based STAP algorithms in [20, 21, 23-29] without prior knowledge of clutter ridge in this paper.

\section{Proposed SASM D3-SR-STAP Algorithm}

To overcome the above two drawbacks of existing sparsitybased STAP algorithms, we propose a novel SASM D3-SRSTAP algorithm in this section. The proposed algorithm uses the snapshot in the CUT as the conventional D3-SRSTAP algorithm does but can obtain better performance than the conventional D3-SR-STAP algorithm by utilizing SASM techniques. Because the proposed algorithm does not need any training snapshots, it can avoid the increase of false alarms caused by discrete interferers only appearing in the CUT, whereas the NSR-STAP can not avoid that in this case.

3.1. Multiple Subsnapshots Generation. To form multiple subsnapshots with the snapshot in the CUT, the SASM techniques are utilized to exploit the space-time structure of the steering vector together with the uncorrelated nature of the components of the interference covariance matrix [5$8,35]$. With $\mathbf{x}$ defined in (3), let $\mathbf{x}_{p, q}$ denote the subsnapshot generated using $N^{\prime}$ consecutive pulses starting from the $p$ th pulse and $M^{\prime}$ antenna elements starting from the $q$ th antenna element (where $1 \leq p \leq N-N^{\prime}+1$ and $1 \leq q \leq M-M^{\prime}+1$ ). This subsnapshot can be realized by a $N^{\prime} M^{\prime} \times N M$ decimation matrix $\mathbf{D}_{p, q}$ with the form of

$$
\mathbf{D}_{p, q}=\left[\mathbf{d}_{p, q, 1}, \mathbf{d}_{p, q, 2}, \ldots, \mathbf{d}_{p, q, N^{\prime} M^{\prime}}\right]^{T},
$$

where

$$
\mathbf{d}_{p, q, l}=[\underbrace{0, \ldots, 0}_{z_{p, q, l}}, 1, \underbrace{0, \ldots, 0}_{N M-z_{p, q, l}-1}]^{T}, \quad l=1, \ldots, N^{\prime} M^{\prime},
$$


where $z_{p, q, l}$ is the number of zeros before the only element equal to one. We can set the value of $z_{p, q, l}$ in three ways, that is, utilizing subtemporal, subspatial, and subspatial-temporal aperture smoothing techniques, respectively, as shown in Figure 2. Suppose that the total number of the subsnapshots is $K$, which is equivalent to

$$
K=\left(N-N^{\prime}+1\right)\left(M-M^{\prime}+1\right)
$$

where $N^{\prime}<N$ and $M^{\prime}=M$ correspond to the case that uses subtemporal aperture smoothing, $N^{\prime}=N$ and $M^{\prime}<M$ correspond to the case that uses subspatial aperture smoothing, $N^{\prime}<N$ and $M^{\prime}<M$ correspond to the case that uses all three types of aperture smoothing, and $N^{\prime}=N$ and $M^{\prime}=$ $M$ correspond to the conventional D3-SR-STAP algorithm presented in [23]. From Figure 2, we observe that the value of $z_{p, q, l}$ can be determined by

$$
z_{p, q, l}=\left(p+\left\lfloor\frac{l}{M^{\prime}}\right\rfloor-1\right) M+q+\bmod \left(l, M^{\prime}\right)-1,
$$

where the brackets \lfloor\rfloor denote the floor operator and mod $\left(l, M^{\prime}\right)=l-\left\lfloor l / M^{\prime}\right\rfloor M^{\prime}$ denotes the modulo operator.

After determining the decimation matrix $\mathbf{D}_{p, q}$, we can obtain the $(p, q)$ th subsnapshot by

$$
\mathbf{x}_{p, q}=\mathbf{D}_{p, q} \mathbf{x}
$$

Note that $\mathbf{x}_{p, q}$ is of the size $N^{\prime} M^{\prime} \times 1$ and can be further expressed as

$$
\begin{aligned}
\mathbf{x}_{p, q} & =\mathbf{D}_{p, q} \boldsymbol{\Phi} \boldsymbol{\gamma}+\mathbf{D}_{p, q} \mathbf{n} \\
& =\sum_{i=1}^{N_{d}} \sum_{k=1}^{N_{s}} \gamma_{i, k} \mathbf{D}_{p, q} \mathbf{v}\left(f_{d, i}, \phi_{k}\right)+\mathbf{n}_{p, q} \\
& =\sum_{i=1}^{N_{d}} \sum_{k=1}^{N_{s}} \bar{\gamma}_{p, q, i, k} \overline{\mathbf{v}}\left(f_{d, i}, \phi_{k}\right)+\mathbf{n}_{p, q},
\end{aligned}
$$

where $\mathbf{n}_{p, q}=\mathbf{D}_{p, q} \mathbf{n}, \bar{\gamma}_{p, q, i, k}=e^{j 2 \pi\left[(p-1) M f_{d, i}+(q-1) f_{s, k}\right]} \gamma_{i, k}$, and

$$
\overline{\mathbf{v}}\left(f_{d, i}, \phi_{k}\right)=\left[\begin{array}{c}
1 \\
\vdots \\
e^{j 2 \pi\left(M^{\prime}-1\right) f_{s, k}} \\
\vdots \\
e^{\left.j 2 \pi\left[l l / M^{\prime}\right] M f_{d, i}+\bmod \left(l-1, M^{\prime}\right) f_{s, k}\right]} \\
\vdots \\
e^{j 2 \pi\left[\left(N^{\prime}-1\right) M f_{d, i}+\left(M^{\prime}-1\right) f_{s, k}\right]}
\end{array}\right],
$$

with $l=1,2, \ldots, N^{\prime} M^{\prime}$. Thus, the generated subsnapshot can be rewritten as

$$
\mathbf{x}_{p, q}=\overline{\boldsymbol{\Phi}} \bar{\gamma}_{p, q}+\mathbf{n}_{p, q}
$$

where

$$
\overline{\boldsymbol{\Phi}}=\left[\overline{\mathbf{v}}\left(f_{d, 1}, \phi_{1}\right), \overline{\mathbf{v}}\left(f_{d, 1}, \phi_{2}\right), \ldots, \overline{\mathbf{v}}\left(f_{d, N_{d}}, \phi_{N_{s}}\right)\right] .
$$

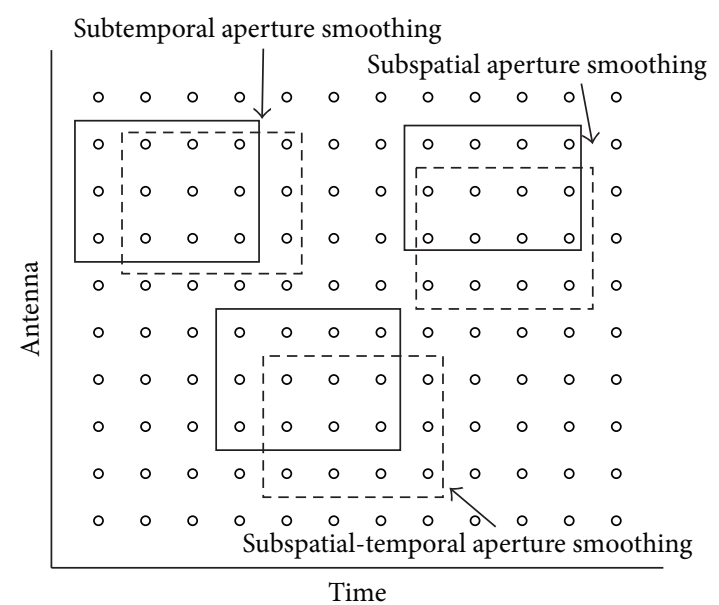

FIGURE 2: Generation of the subsnapshots from the space-time snapshot in the CUT using SASM techniques.

Comparing the formulations between $\bar{\gamma}_{p, q}$ and $\gamma$, we find that there is only a phase difference $e^{j 2 \pi\left[(p-1) M f_{d, i}+(q-1) f_{s, k}\right]}$ among the corresponding elements, which results in $\gamma \odot \gamma^{*}=$ $\bar{\gamma}_{p, q} \odot \bar{\gamma}_{p, q}^{*}$. In other words, different subsnapshots have the same angle-Doppler profile amplitude $|\gamma|$. From the point of sparse recovery, these subsnapshots are called multiple measurement vectors (MMV) and the solutions share a common sparsity profile [37].

3.2. Interference Covariance Matrix Estimation and Filter Design. Based on the above discussions, the interference covariance matrix of the $(p, q)$ th subsnapshot can be written as

$$
\begin{aligned}
\mathbf{R}_{p, q} & =E\left[\mathbf{x}_{p, q} \mathbf{x}_{p, q}^{H}\right] \\
& =\overline{\boldsymbol{\Phi}} E\left[\bar{\gamma}_{p, q} \bar{\gamma}_{p, q}^{H}\right] \overline{\boldsymbol{\Phi}}^{H}+E\left[\mathbf{n}_{p, q} \mathbf{n}_{p, q}^{H}\right] \\
& =\overline{\boldsymbol{\Phi}} E\left[\operatorname{diag}\left(\boldsymbol{\gamma}_{p, q} \odot \gamma_{p, q}^{*}\right)\right] \overline{\boldsymbol{\Phi}}^{H}+\sigma_{n}^{2} \mathbf{I}_{N^{\prime} M^{\prime}} \\
& =\overline{\boldsymbol{\Phi}} E\left[\operatorname{diag}\left(\gamma \odot \gamma^{*}\right)\right] \overline{\boldsymbol{\Phi}}^{H}+\sigma_{n}^{2} \mathbf{I}_{N^{\prime} M^{\prime}} \\
& =\overline{\boldsymbol{\Phi}} \boldsymbol{\Gamma} \overline{\boldsymbol{\Phi}}^{H}+\sigma_{n}^{2} \mathbf{I}_{N^{\prime} M^{\prime}} .
\end{aligned}
$$

In the above derivation, we have used the fact that (i) different elements in the $(p, q)$ th angle-Doppler profile are independent of each other, as well as different elements in the $(p, q)$ th thermal noise vector $\mathbf{n}_{p, q}$; and (ii) $\gamma \odot \gamma^{*}=$ $\bar{\gamma}_{p, q} \odot \bar{\gamma}_{p, q}^{*}$. From (17), we find that different subsnapshots share the same interference covariance matrix. Furthermore, it is noted that the full dimension (with the DOFs of NM) interference covariance matrix can be obtained according to (5) if the parameter $\Gamma=\operatorname{diag}(\mathbf{a})=E\left[\boldsymbol{\gamma} \odot \boldsymbol{\gamma}^{*}\right]$ is known. In other words, although we use the subsnapshots to recover the angle-Doppler profile, we can obtain the interference covariance matrix estimate exactly the same as what we get using the full snapshot. This will lead to no DOFs loss in the proposed algorithm. For the real applications, we can 
not obtain the true parameter a but can estimate it using the generated subsnapshots with the form of

$$
\widehat{\mathbf{a}}=\frac{1}{K} \sum_{p=1}^{N-N^{\prime}+1} \sum_{q=1}^{M-M^{\prime}+1} \bar{\gamma}_{p, q} \odot \bar{\gamma}_{p, q}^{*}
$$

There are several aspects which should be noted about the recovery of angle-Doppler profile $\bar{\gamma}_{p, q}$ in (18). First, the values of $N^{\prime}$ and $M^{\prime}$ not only decide the number of subsnapshots but also are related to the accuracy of the recovered angleDoppler profile. However, there is no theoretic argument in current literature to determine how large the size of the subsnapshot could be to obtain a unique recovery of the angle-Doppler profile, since the columns of the space-time steering dictionary $\overline{\boldsymbol{\Phi}}$ are highly correlated and the samples are contaminated by the thermal noise. The following simulations will show that there is such a range of $N^{\prime}$ and $M^{\prime}$ that the angle-Doppler profiles will be well recovered from the subsnapshots. Second, since the convergence of the sparsitybased STAP algorithms is very fast (e.g., a good estimation of the interference covariance matrix can be obtained with the number of subsnapshots $K$ equal to 3 or $4[25,26])$, the size of $N^{\prime} M^{\prime}$ can be slightly smaller than $N M$. It is said that we can employ negligible loss in system DOFs to obtain multiplicative increment in the snapshots.

Thirdly, the subsnapshots are generated only using the received data in the CUT. On one hand, all subsnapshots satisfy a common sparsity profile, which is very important especially in presence of discrete interferers merely in the CUT. In this case, the snapshots adjacent to the CUT will have different sparsity profiles with the snapshot in the CUT, which results in significant bias between the estimated interference covariance matrix and the true interference covariance matrix of the CUT. On the other hand, because the recovered angle-Doppler profile in the CUT may also contain the information of target, it is necessary to eliminate the impacts of the target signal present in the CUT. This can be performed as the approach described in [23], which first determines the signal of interest area $\Omega$ in the angle-Doppler plane using the prior knowledge of the target signal (it is usually assumed to be known for STAP problem) as

$$
\Omega=\left\{\left(i_{n_{1}}, k_{n_{1}}\right),\left(i_{n_{2}}, k_{n_{2}}\right), \ldots,\left(i_{n_{N_{\mathrm{SOI}}}}, k_{n_{\mathrm{N}_{\mathrm{SOI}}}}\right)\right\},
$$

where $\left(i_{n_{m}}, k_{n_{m}}\right), 1 \leq m \leq N_{\text {SOI }}$ denote the possible indexes of the target signal in the discretized angle-Doppler plane. The size of $N_{\text {SOI }}$ reflects the uncertainty along the angle and Doppler frequency axes. Then, the target signal is eliminated from the estimated interference spectrum by marking zero coefficients corresponding to $\Omega$ in the recovered angleDoppler profile $\bar{\gamma}_{p, q}$.

After that, the interference plus noise covariance matrix can be estimated by

$$
\widehat{\mathbf{R}}=\sum_{i=1, k=1}^{N_{d}, N_{s}} \widehat{a}_{i, k} \mathbf{v}\left(f_{d, i}, \phi_{k}\right) \mathbf{v}^{H}\left(f_{d, i}, \phi_{k}\right)+\widehat{\sigma}_{n}^{2} \mathbf{I}, \quad i, k \notin \Omega,
$$

where $\widehat{\sigma}_{n}^{2}$ is the estimated thermal noise power level.
After we obtain the estimated clutter plus noise covariance matrix, the idea behind linear constraint minimum variance (LCMV) approach is to minimize the interference plus noise output power whilst constraining the gain in the direction of the desired signal, which leads to the following minimization with constraints:

$$
\min _{\mathbf{w}} \mathbf{w}^{H} \widehat{\mathbf{R}} \mathbf{w} \quad \text { s.t. } \mathbf{w}^{H} \mathbf{s}=1 \text {, }
$$

where $\mathbf{s}=\mathbf{v}\left(f_{d, t}, \phi_{t}\right)$ represents the space-time steering vector in the target direction $\left(f_{d, t}\right.$ and $\phi_{t}$ are the target Doppler frequency and the target AOA, resp.). Using the method of Lagrange multipliers, the LCMV STAP filter weight vector is given by

$$
\widehat{\mathbf{w}}=\frac{\widehat{\mathbf{R}}^{-1} \mathbf{s}}{\mathbf{s}^{H} \widehat{\mathbf{R}}^{-1} \mathbf{s}} .
$$

Thus, the target can be detected by the adaptive matched filter (AMF) detector with the form

$$
\frac{\left|\mathbf{s}^{H} \widehat{\mathbf{R}}^{-1} \mathbf{x}\right|^{2}}{\mathbf{s}^{H} \widehat{\mathbf{R}}^{-1} \mathbf{s}} \underset{H_{0}}{\stackrel{H_{1}}{\gtrless}} \xi_{\mathrm{AMF}},
$$

where $H_{0}$ is the null hypothesis (i.e., target absence), $H_{1}$ is the alternative hypothesis (i.e., target presence), and $\xi_{\mathrm{AMF}}$ represents the target detection threshold.

It is remarked that the conventional D3-LS STAP methods [5-8] firstly eliminate the target signal effects and then employ the SASM technique to generate a number of subsnapshots. The proposed algorithm directly employs the SASM technique for the received data in the CUT to generate multiple subsnapshots and uses these subsnapshots to recover the interference spectrum. After determining the target of interest area $\Omega$ in the angle-Doppler plane using the prior knowledge, the target signal can be eliminated from the estimated interference spectrum by marking zero coefficients corresponding to $\Omega$ in the recovered angle-Doppler profile. This approach will obtain better accuracy of the estimated interference spectrum than the method that eliminates the target signal before sparse recovery. This is because of the fact that if we eliminate the target signal before sparse recovery as in the D3-LS approach, some interference components correlated with the target signal will also be canceled. On the other hand, if we directly recover the interference spectrum, one can expect to obtain simultaneously good estimation of the interference components and the target signals, for example, the recovered results in [23].

3.3. Complexity Analysis. For the proposed algorithm, the computational complexity mainly comes from the recovery of the angle-Doppler profile $\bar{\gamma}_{p, q}$. If we use the SR algorithms with single measurement vector (SMV-SR) to recover $\bar{\gamma}_{p, q}$ one by one, the computational complexity will be $K$ times of that of recovering single angle-Doppler profile. This will lead to very high computational complexity. However, if we use the SR algorithms with MMV (MMV-SR) [37], the computational complexity will be significantly reduced compared with that of recovering the angle-Doppler profiles one by one. 
TABLE 1: Radar system parameters.

\begin{tabular}{lc}
\hline Parameter & Value \\
\hline Antenna array & side-looking ULA \\
Antenna array spacing & $\lambda_{c} / 2$ \\
Number of elements in ULA & 12 \\
Number of pulses in one CPI & 12 \\
Carrier wavelength & $\lambda_{c}=0.3 \mathrm{~m}$ \\
Instantaneous bandwidth & $5 \mathrm{MHz}$ \\
Transmit taper & Uniform \\
Pulse repetition frequency (PRF) & $4000 \mathrm{~Hz}$ \\
Platform velocity & $300 \mathrm{~m} / \mathrm{s}$ \\
Platform height & $3000 \mathrm{~m}$ \\
Clutter-to-noise ratio (CNR) & $30 \mathrm{~dB}$ \\
The target AOA & $0^{\circ}$ \\
\hline
\end{tabular}

Of course, the computational complexity of the MMV-SR algorithms is still higher than that of recovering single angleDoppler profile.

\section{Simulation Results}

In this section, we assess the output SINR and probability of detection $\left(P_{d}\right)$ performance of the proposed algorithm using simulated data and compare it with the generalized $\mathrm{F} / \mathrm{B}$ method in [35], the NSR-STAP algorithm in [25, 26], the D3LS STAP method in [7], and the D3-SR-STAP algorithm in [23]. The parameters of the airborne radar systems are shown in Table 1. The thermal noise is modeled as a Gaussian white noise with unity power. The clutter and target powers are referred to as the thermal noise power. In the simulations, the diagonal loading factor $\widehat{\sigma}_{n}^{2}$ is set to the noise level for all algorithms. For the sparsity-based STAP algorithms, we set $\rho_{d}=\rho_{s}=4$ and use recently developed fast sparse recovery algorithm, called focal underdetermined system solution (FOCUSS) $[37,38]$, with the same parameters setting to compute the angle-Doppler profiles. For the NSR-STAP algorithm and the generalized F/B method, the numbers of training snapshots are set to 4 and 30, respectively. In addition, $N^{\prime}=11$ and $M^{\prime}=11$ for the generalized $\mathrm{F} / \mathrm{B}$ method and $N^{\prime}=8$ and $M^{\prime}=8$ for the D3-LS STAP method. The output SINR loss is defined to be its output SINR performance relative to the matched filter $\mathrm{SNR}_{\mathrm{opt}}$ in an interference-free environment [1], given as

$$
\operatorname{SINR}=\frac{\left|\widehat{\mathbf{w}}^{H} \mathbf{s}\right|^{2}}{\operatorname{SNR}_{\text {opt }}\left|\widehat{\mathbf{w}}^{H} \mathbf{R} \widehat{\mathbf{w}}\right|}=\frac{\left|\widehat{\mathbf{w}}^{H} \mathbf{s}\right|^{2}}{N M\left|\widehat{\mathbf{w}}^{H} \mathbf{R} \widehat{\mathbf{w}}\right|},
$$

where $\mathbf{R}$ is the true interference covariance matrix.

4.1. Setting of the Size of $N^{\prime} M^{\prime}$. In this subsection, we investigate the selection of the size of $N^{\prime} M^{\prime}$ in the proposed SASM D3-SR-STAP algorithm. In the example, consider homogeneous environments with none discrete interferers and the target with the AOA $0^{\circ}$ and normalized Doppler frequency 0.25 . When $N^{\prime}<N$ and $M^{\prime}=M$, the subsnapshots are only

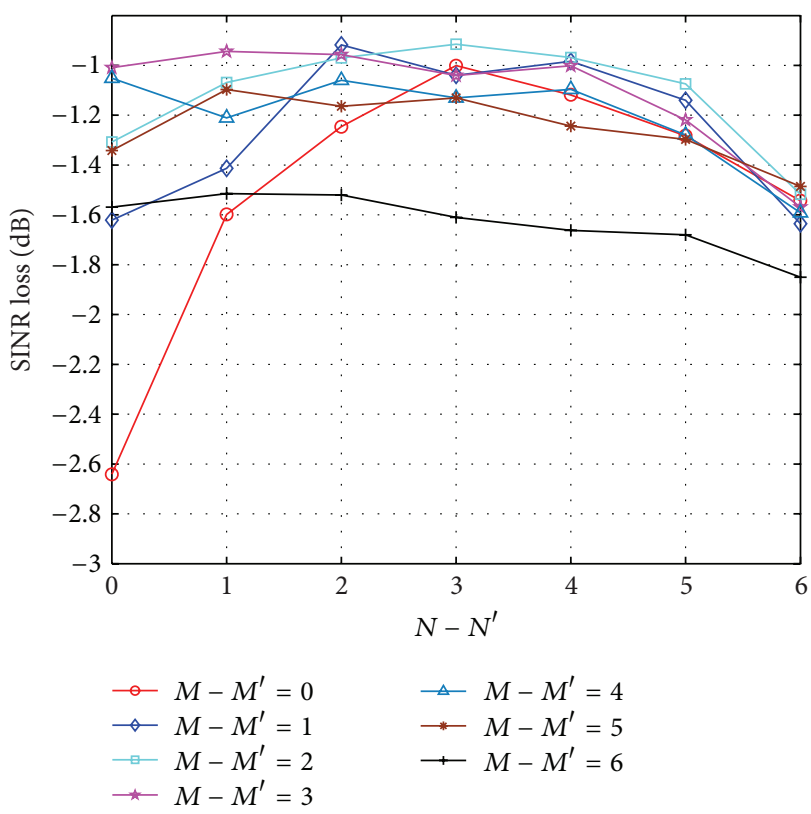

FIgURE 3: SINR loss versus the sizes of $N-N^{\prime}$ and $M-M^{\prime}$.

generated using the subtemporal smoothing technique; when $N^{\prime}=N$ and $M^{\prime}<M$, the subsnapshots are only generated using the subspatial smoothing technique; when $N^{\prime}<N$ and $M^{\prime}<M$, the subsnapshots are generated by the subtemporal smoothing, subspatial smoothing, and subspatial-temporal smoothing techniques. The simulation results are plotted in Figure 3. It is seen from the curves that (i) the output SINR performance of the proposed SASM D3-SR-STAP algorithm is better than that of the conventional D3-SR-STAP algorithm corresponding to the case of $N^{\prime}=N$ and $M^{\prime}=M$ since the proposed algorithm uses multiple subsnapshots to estimate the interference covariance matrix. (ii) There is a range of the sizes of $N^{\prime}$ and $M^{\prime}$ to obtain a good SINR performance, which leads to a relaxing parameter setting of $N^{\prime}$ and $M^{\prime}$. (iii) When $M-M^{\prime}>5$ or $N-N^{\prime}>5$, the performance of the proposed algorithm degrades because of too many DOFs reduced (corresponding to not enough observation data to recover the angle-Doppler profile). Therefore, for our simulations, it is best to select the size of $N^{\prime} M^{\prime}$ by making the ratio of $\left(N^{\prime} M^{\prime}\right) /(N M)$ as big as possible whilst keeping $K=\left(N-N^{\prime}+1\right)\left(M-M^{\prime}+1\right)>3$.

4.2. Comparison with Existing Algorithms. In the first example, we focus on the impact of discrete interferers only in presence of the CUT to the proposed algorithm. Consider two different cases: case 1, the homogeneous environments with none discrete interferers where the ideal spectral distribution of the interference is a diagonal line in the angle-Doppler plane; case 2, the nonhomogeneous environments. In this case, the snapshot in the CUT is a sum of clutter, one discrete interferer (the $\mathrm{AOA}-30^{\circ}$, the normalized Doppler frequency 0.3 , and the power $25 \mathrm{~dB}$ ), and the thermal noise, while the snapshots adjacent to the CUT are only a sum of clutter and thermal noise. Thus, the spectral distribution of the 
snapshots adjacent to the CUT is different from that of the snapshot in the CUT, where the ideal spectral distribution of the interference in the CUT is the clutter ridge plus the discrete interferers. In the example, according to the results of the above subsection, we set $M^{\prime}=M$ and $N-N^{\prime}=3$ for the proposed algorithm. The results that plot the SINR loss performance against the target Doppler frequency of the proposed algorithm and the existing algorithms are shown in Figures 4 and 5, which are averaged over 100 independent Monte Carlo runs. In Figure 5, the "No discrete interferer" denotes the approach that uses the precise clutter plus noise covariance matrix without discrete interferers information.

From the figures, we see that the proposed SASM D3-SRSTAP algorithm, the conventional D3-LS-STAP algorithm, and the D3-SR-STAP algorithm are robust to the case that the discrete interferers merely appear in the CUT, but the performance of the generalized F/B method, the NSR-STAP algorithm, and the approach of "No discrete interferer" is significantly degraded in this case. This is because the estimated covariance matrices that use the generalized F/B method, the NSR-STAP algorithm, and the approach of "No discrete interferer" do not include the discrete interferers information and can not effectively suppress the discrete interferers in the CUT. The D3 STAP algorithms (like the proposed SASM D3-SR-STAP algorithm, the conventional D3-LS-STAP algorithm, and the D3-SR-STAP algorithm) use only the snapshot in the CUT, thereby including both the clutter and discrete interferers information, and can suppress both the clutter and discrete interferers. We also observe that the proposed SASM D3-SR-STAP algorithm outperforms the generalized F/B method, the conventional D3-LS-STAP algorithm, and the D3-SR-STAP algorithm in most of Doppler bins in both cases but performs slightly worse than the generalized F/B method in the Doppler range of -0.1 to 0.1 . Although the proposed SASM D3-SR-STAP algorithm achieves worse SINR performance than the NSR-STAP algorithm in homogeneous environments, it obtains a much better SINR performance in nonhomogeneous environments. The cause for this is that the NSR-STAP algorithm uses more observed snapshots than the proposed algorithm and can achieve better performance than the proposed algorithm in homogeneous environments. However, in nonhomogeneous environments, the NSR-STAP algorithm does have a good estimation of the clutter covariance matrix, but it has no information of the discrete interferers resulting in a significant performance reduction.

In the second example, we focus on the impact of model mismatch, such as the ICM and the channel mismatch, to the proposed SASM D3-SR-STAP algorithm. The ICM can be formulated as a general model proposed by Ward in [1], which is suitable over the water scenario. The temporal autocorrelation of the fluctuations is Gaussian in shape with the form

$$
\zeta(m)=\exp \left\{-\frac{8 \pi^{2} \sigma_{v}^{2} T_{r}^{2}}{\lambda_{c}^{2}} m^{2}\right\}
$$

where $T_{r}$ is the pulse repetition interval and $\sigma_{v}$ is the velocity standard deviation (in the example, we set $\sigma_{v}=1$ corresponding to a moderate clutter Doppler spreading situation).

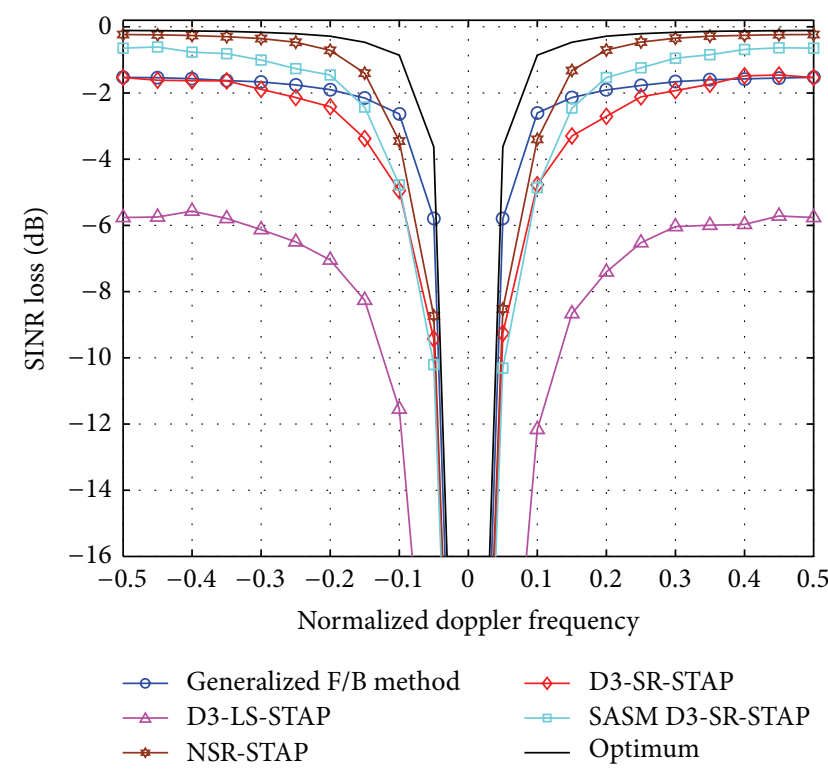

FIGURE 4: SINR loss against the target Doppler frequency in homogeneous environments.

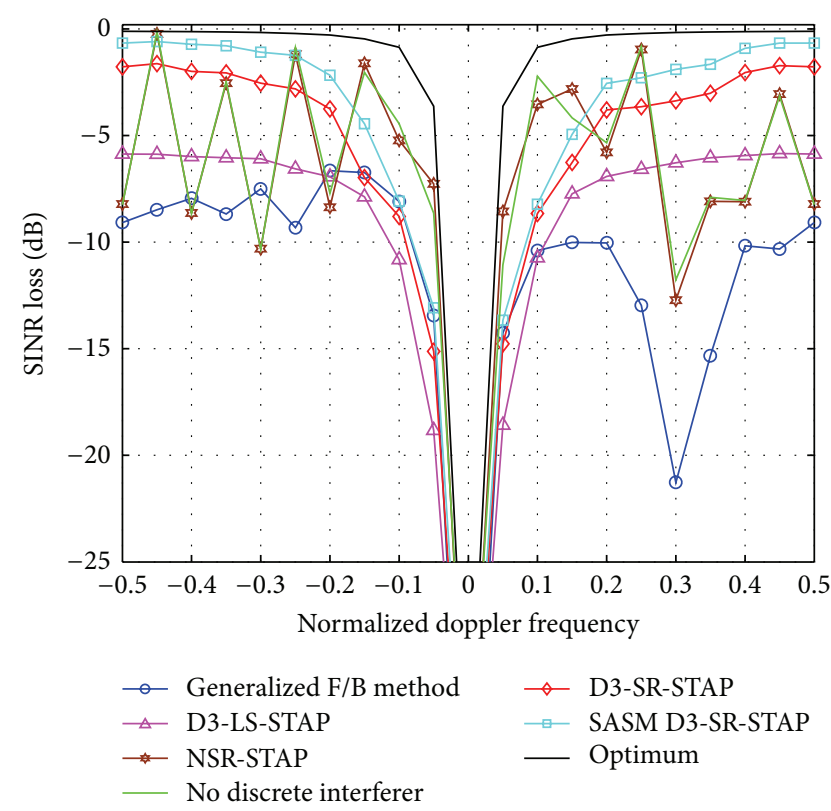

FIgURE 5: SINR loss against the target Doppler frequency in nonhomogeneous environments.

With regard to the channel mismatch, we only consider the angle-independent array errors described in [2]; that is, the amplitude and phase errors are modeled as a narrowband case as follows:

$$
\begin{aligned}
p\left(\delta_{\epsilon_{a}}\right) & = \begin{cases}\frac{1}{\Delta \epsilon_{a}}, & \text { for } 0 \leq \delta_{\epsilon_{a}} \leq \Delta \epsilon_{a} \\
0, & \text { elsewhere, }\end{cases} \\
p\left(\delta_{\epsilon_{p}}\right) & = \begin{cases}\frac{1}{\Delta \epsilon_{p}}, & \text { for }-\frac{\Delta \epsilon_{p}}{2} \leq \delta_{\epsilon_{p}} \leq \frac{\Delta \epsilon_{p}}{2} \\
0, & \text { elsewhere }\end{cases}
\end{aligned}
$$




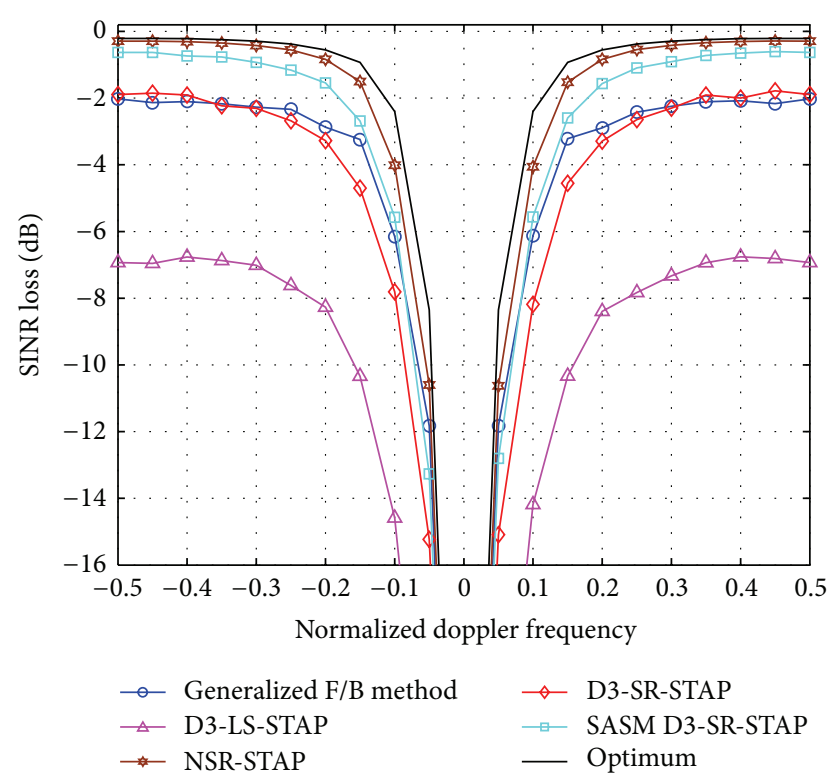

FIgURE 6: SINR loss against the target Doppler frequency considering ICM.

where $p\left(\delta_{\epsilon_{a}}\right)$ and $p\left(\delta_{\epsilon_{p}}\right)$ are the probability density functions (pdfs) (uniform) associated with the amplitude and phase errors, respectively. The sparsity-based STAP algorithms can be easily extended to the situation in which the array has been calibrated. In fact, we only have to modify the spacetime steering vectors in the space-time steering dictionary. Therefore, in the example, we set $\Delta \epsilon_{a}=0.01$ and $\Delta \epsilon_{p}=2^{\circ}$ corresponding to the general case after the array has been calibrated [16]. In severe channel mismatch cases, the array must be calibrated before using the proposed approach. The general calibration-on-clutter methods are summarized in Table 3 in [16]. Interested readers are referred to [16] for details. The other parameters are the same as those in Table 1.

Figures 6 and 7 depict the SINR loss performance against the target Doppler frequency of all algorithms under the cases considering the ICM and the channel mismatch, respectively. Compared with the case without ICM and channel mismatch in Figure 4, the curves show that the performance of all algorithms have some degradations because of the ICM and the channel mismatch. However, the performance of the proposed algorithm is still better than the generalized F/B method, the conventional D3-LS-STAP algorithm, and the D3-SR-STAP algorithm. Although the proposed algorithm performs worse than the NSR-STAP algorithm, it will not suffer performance decrease in presence of discrete interferers only in the CUT. In addition, the NSR-STAP algorithm requires the training snapshots to satisfy independent and identical distribution resulting in snapshots selection problem, whereas the proposed algorithm can avoid this problem. Furthermore, we present the $P_{d}$ versus the target signal-tonoise ratio (SNR) for all algorithms considering different cases in Figure 8 (the ideal case with no ICM and channel mismatch), Figure 9 (the case with ICM), and Figure 10 (the case with channel mismatch). The false alarm rate $P_{f a}$ is

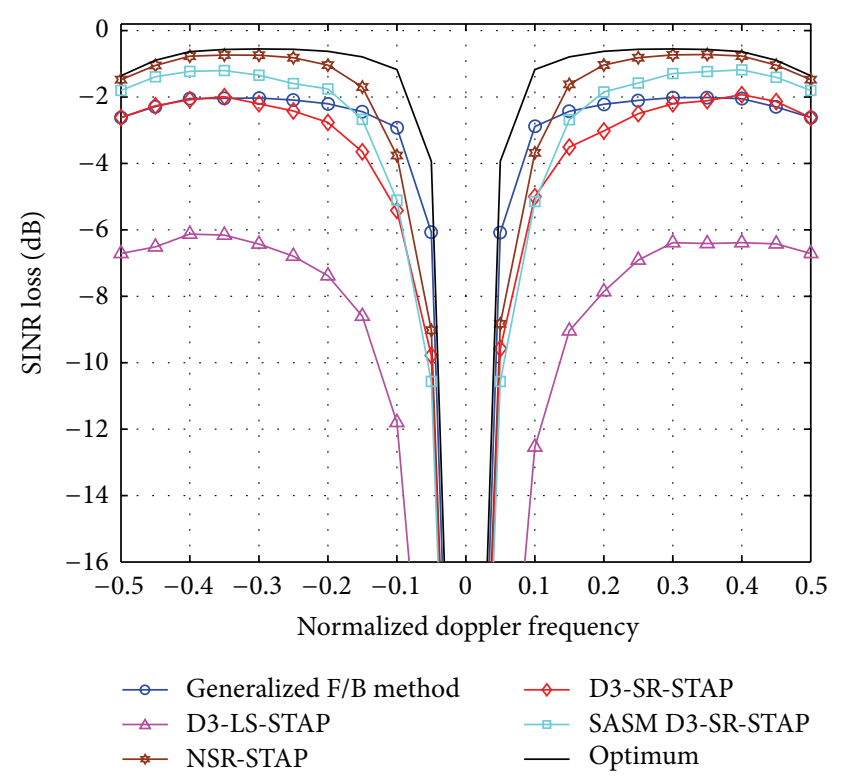

FIGURE 7: SINR loss against the target Doppler frequency considering channel mismatch.

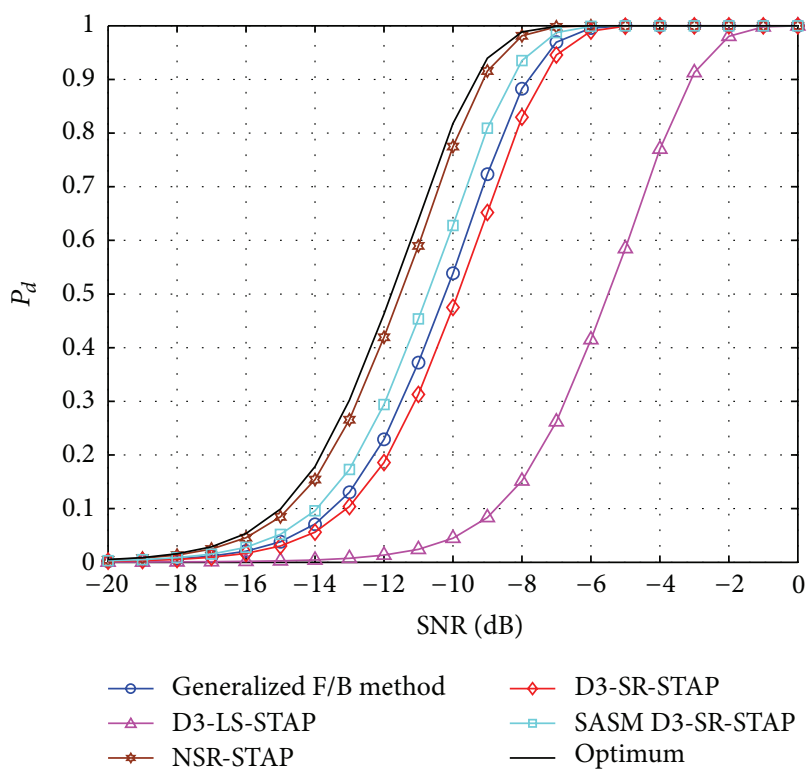

FIgURE 8: $P_{d}$ versus the target SNR in the ideal case.

set to $10^{-4}$ and for simulation purposes the threshold and $P_{d}$ estimates are based on 10,000 samples. We suppose the target is injected in the boresight with normalized Doppler frequency 0.25 . The figure illustrates that the proposed algorithm provides higher detection rate than the generalized F/B method, the conventional D3-LS-STAP algorithm, and the D3-SR-STAP algorithm in all three cases, but a slightly lower detection rate than the NSR-STAP algorithm. However, as one can expect, the proposed algorithm will obtain better detection rate when the discrete interferers merely appear in the CUT. 

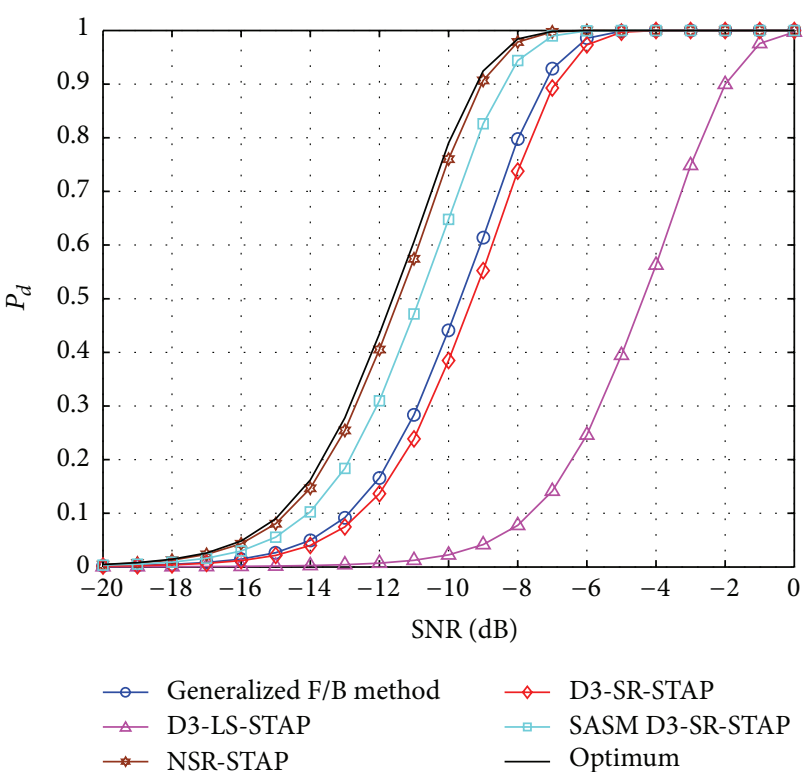

FIgURE 9: $P_{d}$ versus the target SNR considering ICM.

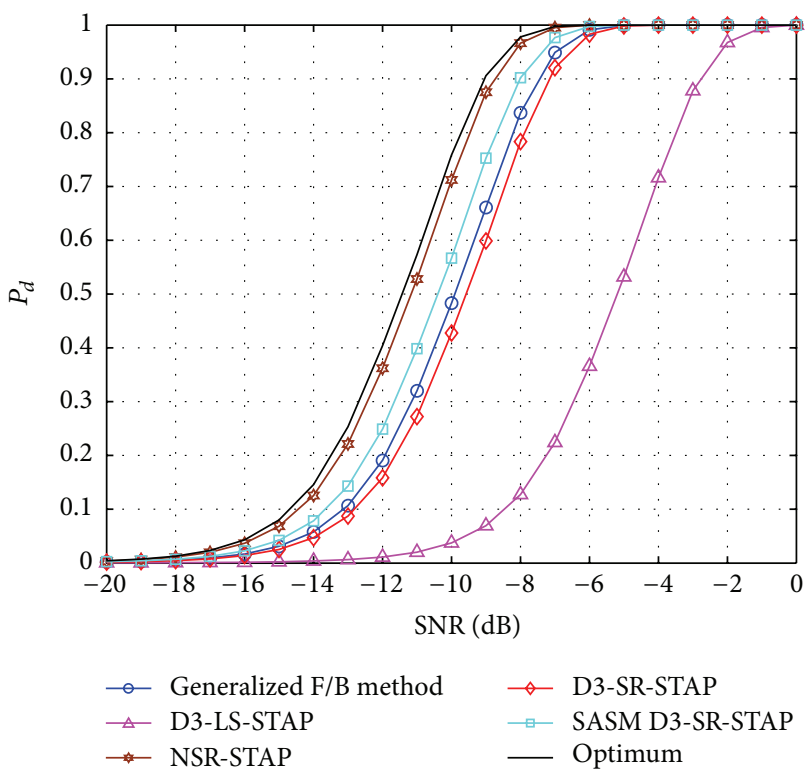

FIgURE 10: $P_{d}$ versus the target SNR considering channel mismatch.

\section{Conclusions}

In this paper, we proposed a novel D3-SR-STAP algorithm utilizing the SASM techniques for airborne radar applications. Different from either NSR-STAP or D3-SR-STAP, the proposed algorithm generates multiple subsnapshots with the snapshot in the CUT by exploiting the space-time structure of the steering vector and the uncorrelated nature of the components of the interference covariance matrix. By jointly using these multiple subsnapshots and the sparsity of interference spectrum in the angle-Doppler plane, the proposed algorithm can obtain a better interference spectrum estimate, thereby a higher output SINR, than the D3-SR-STAP algorithm.
Simulation results have shown that the proposed algorithm outperforms the generalized F/B method, the conventional D3-LS-STAP algorithm, and the D3-SR-STAP algorithm in both homogeneous and nonhomogeneous environments and provides better performance than the NSR-STAP algorithm in presence of discrete interferers merely in the CUT. Since the model mismatch is only considered in simulations in the paper, further investigation about the theoretical analysis of the model mismatch impacts on sparsity-based STAP algorithms is required.

\section{Conflict of Interests}

The authors declare that there is no conflict of interests regarding the publication of this paper.

\section{Acknowledgment}

This work is funded in part by the National Natural Science Foundation of China (61101182, 61201334, and 61401478).

\section{References}

[1] J. Ward, "Space-time adaptive processing for airborne radar," Technical Report 1015, MIT Lincoln laboratory, Lexington, Mass, USA, 1994.

[2] J. R. Guerci, Space-Time Adaptive Processing for Radar, Artech House, 2003.

[3] W. L. Melvin, "A STAP overview," IEEE Aerospace and Electronic Systems Magazine, vol. 19, no. 1, pp. 19-35, 2004.

[4] R. Klemm, Principles of Space-Time Adaptive Processing, Institute of Electrical Engineering, London, UK, 2006.

[5] T. K. Sarkar and N. Sangruji, "Adaptive nulling system for a narrow-band signal with a look-direction constraint utilizing the conjugate gradient method," IEEE Transactions on Antennas and Propagation, vol. 37, no. 7, pp. 940-944, 1989.

[6] H. Wang, Y. Zhang, and Q. Zhang, "View of current status of space-time processing algorithms research," in Proceedings of the IEEE International Radar Conference, pp. 635-640, Alexandria, Va, USA, May 1995.

[7] T. K. Sarkar, H. Wang, S. Park et al., "A deterministic leastsquares approach to space-time adaptive processing (STAP)," IEEE Transactions on Antennas and Propagation, vol. 49, no. 1, pp. 91-103, 2001.

[8] D. Cristallini and W. Burger, "A robust direct data domain approach for STAP," IEEE Transactions on Signal Processing, vol. 60, no. 3, pp. 1283-1294, 2012.

[9] E. Aboutanios and B. Mulgrew, "Hybrid detection approach for STAP in heterogeneous clutter," IEEE Transactions on Aerospace and Electronic Systems, vol. 46, no. 3, pp. 1021-1033, 2010.

[10] E. Yang, R. Adve, J. Chun, and J. Chun, "Hybrid direct data domain sigma-delta space-time adaptive processing algorithm in non-homogeneous clutter," IET Radar, Sonar and Navigation, vol. 4, no. 4, pp. 611-625, 2010.

[11] C. T. Capraro, G. T. Capraro, I. Bradaric, D. D. Weiner, M. C. Wicks, and W. J. Baldygo, "Implementing digital terrain data in knowledge-aided space-time adaptive processing," IEEE Transactions on Aerospace and Electronic Systems, vol. 42, no. 3, pp. 1080-1097, 2006. 
[12] A. Farina, P. Lombardo, and M. Pirri, "Nonlinear nonadaptive space-time processing for airborne early warning radar," IEE Proceedings-Radar, Sonar and Navigation, vol. 145, no. 1, pp. 918, 1998.

[13] A. Farina, P. Lombardo, and M. Pirri, "Nonlinear STAP processing," Electronics \& Communication Engineering Journal, vol. 11, no. 1, pp. 41-48, 1999.

[14] K. Gerlach and M. L. Picciolo, "Airborne/spacebased radar STAP using a structured covariance matrix," IEEE Transactions on Aerospace and Electronic Systems, vol. 39, no. 1, pp. 269-281, 2003.

[15] J. S. Bergin, C. M. Teixeira, P. M. Techau, and J. R. Guerci, "Improved clutter mitigation performance using knowledgeaided space-time adaptive processing," IEEE Transactions on Aerospace and Electronic Systems, vol. 42, no. 3, pp. 997-1009, 2006.

[16] W. L. Mevin and G. A. Showman, "An approach to knowledgeaided covariance estimation," IEEE Transactions on Aerospace and Electronic Systems, vol. 42, no. 3, pp. 1021-1042, 2006.

[17] Z. Yang, R. C. de Lamare, X. Li, and Q. Wang, "Knowledge-aided STAP using low rank and geometry properties," International Journal of Antennas and Propagation, vol. 2014, Article ID 196507, 14 pages, 2014.

[18] W. Xie, K. Duan, F. Gao, Y. Wang, and Z. Zhang, "Clutter suppression for airborne phased radar with conformal arrays by least squares estimation," Signal Processing, vol. 91, no. 7, pp. 1665-1669, 2011.

[19] W. L. Melvin and J. R. Guerci, "Knowledge-aided signal processing: a new paradigm for radar and other advanced sensors," IEEE Transactions on Aerospace and Electronic Systems, vol. 42, no. 3, pp. 983-995, 2006.

[20] S. Maria and J.-J. Fuchs, "Application of the global matched filter to stap data an efficient algorithmic approach," in Proceedings of the IEEE International Conference on Acoustics, Speech and Signal Processing (ICASSP '06), May 2006.

[21] J. Li, X. Zhu, P. Stoica, and M. Rangaswamy, "High resolution angle-doppler imaging for MTI radar," IEEE Transactions on Aerospace and Electronic Systems, vol. 46, no. 3, pp. 1544-1556, 2010.

[22] I. W. Selesnick, S. U. Pillai, K. Y. Li, and B. Himed, "AngleDoppler processing using sparse regularization," in Proceedings of the IEEE International Conference on Acoustics, Speech, and Signal Processing (ICASSP '10), pp. 2750-2753, Dallas, Tex, USA, March 2010.

[23] K. Sun, H. Meng, Y. Wang, and X. Wang, "Direct data domain STAP using sparse representation of clutter spectrum," Signal Processing, vol. 91, no. 9, pp. 2222-2236, 2011.

[24] Z. Yang, X. Li, and H. Wang, "Space-time adaptive processing based on weighted regularized sparse recovery," Progress in Electromagnetics Research B, vol. 42, pp. 245-262, 2012.

[25] K. Sun, H. Zhang, G. Li, H. Meng, and X. Wang, "A novel STAP algorithm using sparse recovery technique," in Proceedings of the IEEE International Geoscience and Remote Sensing Symposium (IGARSS '09), pp. 336-339, July 2009.

[26] Z. Yang, X. Li, H. Wang, and L. Nie, "Sparsity-based space-time adaptive processing using complex-valued homotopy technique for airborne radar," IET Signal Processing, vol. 8, no. 5, pp. 552564, 2014.

[27] Z. Yang, X. Li, H. Wang, and W. Jiang, "On clutter sparsity analysis in space-time adaptive processing airborne radar," IEEE Geoscience and Remote Sensing Letters, vol. 10, no. 5, pp. 12141218, 2013.
[28] Z. Yang, X. Li, H. Wang, and W. Jiang, "Adaptive clutter suppression based on iterative adaptive approach for airborne radar," Signal Processing, vol. 93, no. 12, pp. 3567-3577, 2013.

[29] S. Sen, "OFDM radar space-time adaptive processing by exploiting spatio-temporal sparsity," IEEE Transactions on Signal Processing, vol. 61, no. 1, pp. 118-130, 2013.

[30] Z. Yang, R. C. de Lamare, and X. Li, " $L_{1}$-regularized STAP algorithms with a generalized sidelobe canceler architecture for airborne radar," IEEE Transactions on Signal Processing, vol. 60, no. 2, pp. 674-686, 2012.

[31] Z. Yang, R. C. de Lamare, and X. Li, "Sparsity-aware space-time adaptive processing algorithms with $L_{1}$-norm regularisation for airborne radar," IET Signal Processing, vol. 6, no. 5, pp. 413-423, 2012.

[32] R. C. de Lamare and R. Sampaio-Neto, "Adaptive reduced-rank processing based on joint and iterative interpolation, decimation, and filtering," IEEE Transactions on Signal Processing, vol. 57, no. 7, pp. 2503-2514, 2009.

[33] R. Fa, R. C. de Lamare, and L. Wang, "Reduced-rank STAP schemes for airborne radar based on switched joint interpolation, decimation and filtering algorithm," IEEE Transactions on Signal Processing, vol. 58, no. 8, pp. 4182-4194, 2010.

[34] P. Stoica and R. Moses, Spectral Analysis of Signals, Prentice Hall, Upper Saddle River, NJ, USA, 2005.

[35] S. U. Pillai, Y. L. Kim, and J. R. Guerci, "Generalized forward/backward subaperture smoothing techniques for sample starved STAP," IEEE Transactions on Signal Processing, vol. 48, no. 12, pp. 3569-3574, 2000.

[36] G. W. Titi and D. F. Marshall, “The ARPA/NAVY mountain program: adaptive signal processing for airborne early warning radar," in Proceedings of the IEEE International Conference on Acoustics, Speech, and Signal Processing (ICASSP '96), pp. 11651168, May 1996.

[37] S. F. Cotter, B. D. Rao, K. Engan, and K. Kreutz-Delgado, "Sparse solutions to linear inverse problems with multiple measurement vectors," IEEE Transactions on Signal Processing, vol. 53, no. 7, pp. 2477-2488, 2005.

[38] I. F. Gorodnitsky and B. D. Rao, "Sparse signal reconstruction from limited data using FOCUSS: a re-weighted minimum norm algorithm," IEEE Transactions on Signal Processing, vol. 45, no. 3, pp. 600-616, 1997. 

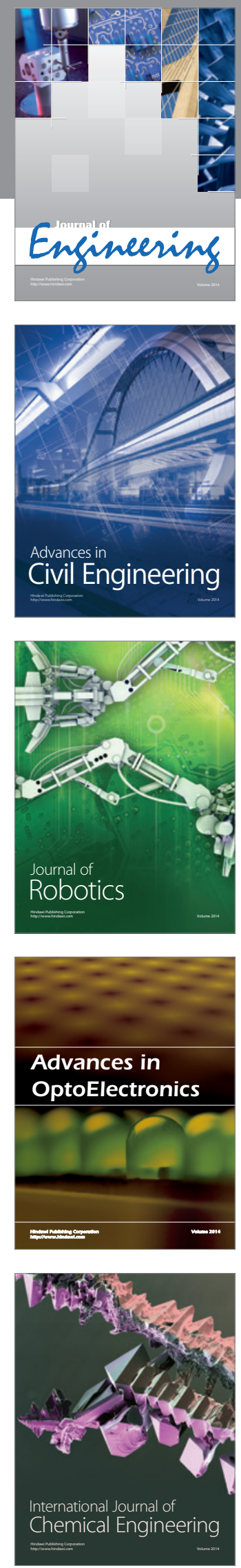

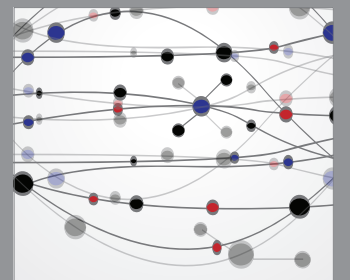

The Scientific World Journal
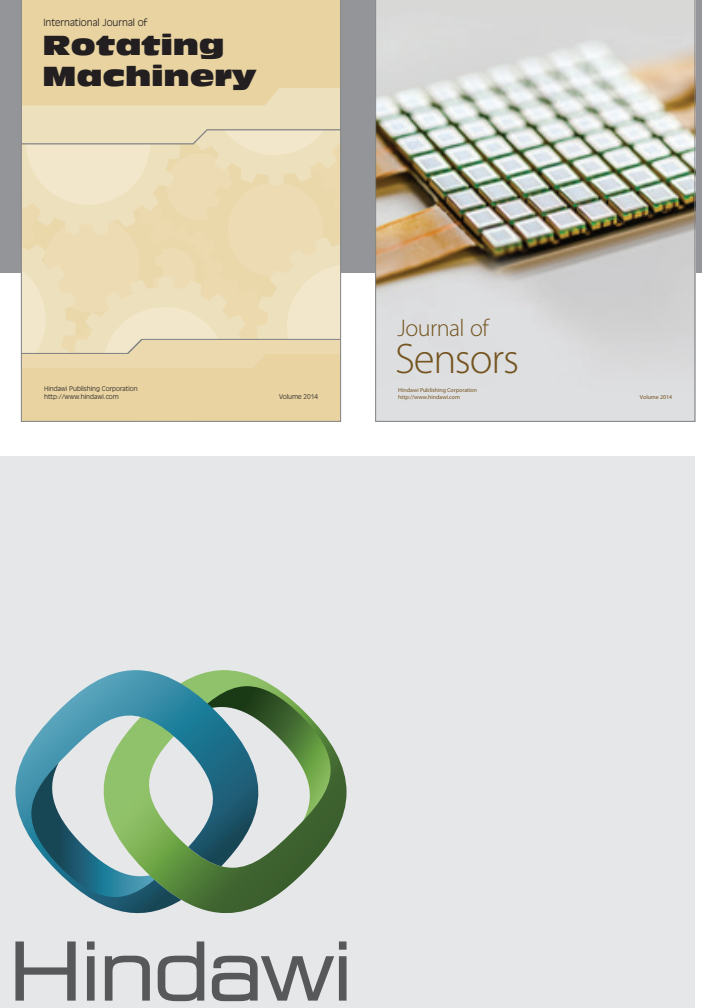

Submit your manuscripts at http://www.hindawi.com
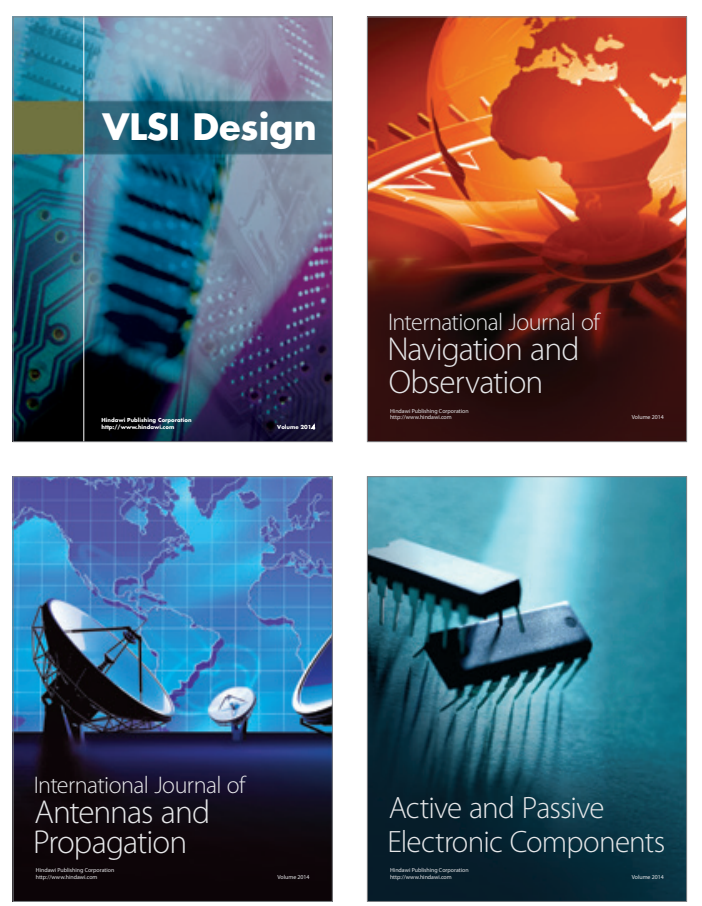
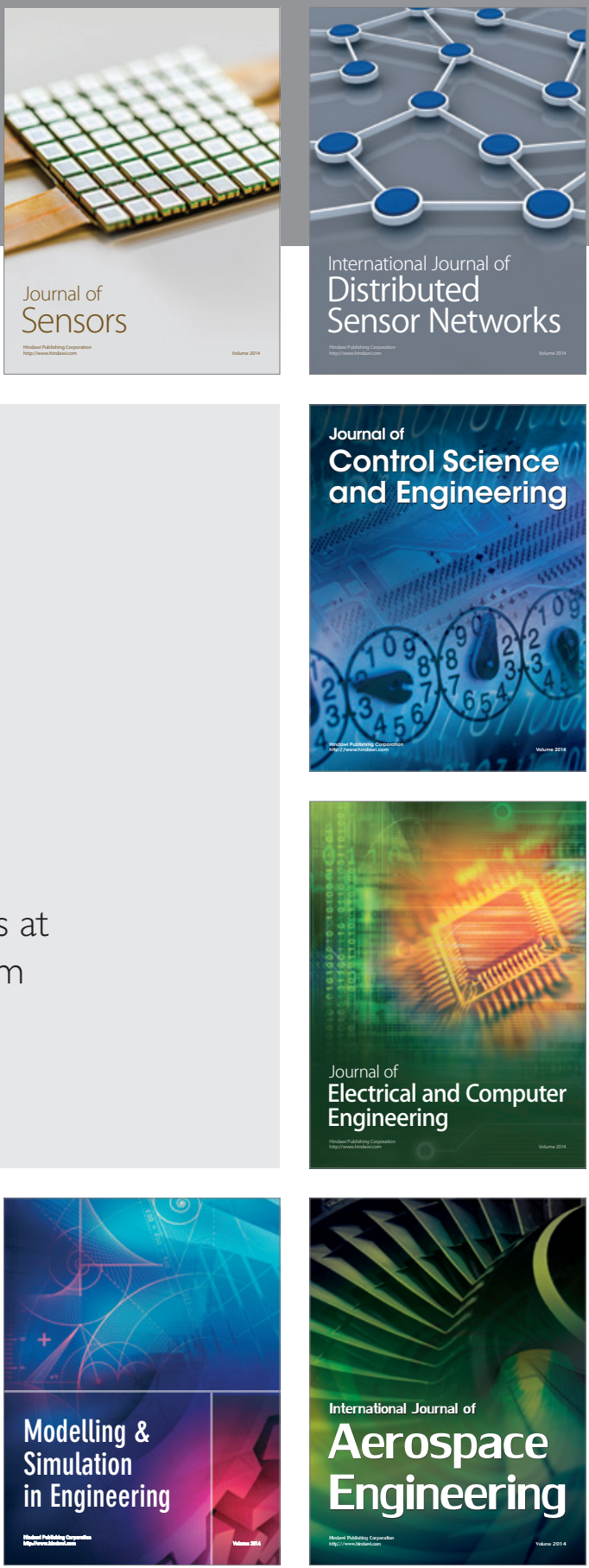

Journal of

Control Science

and Engineering
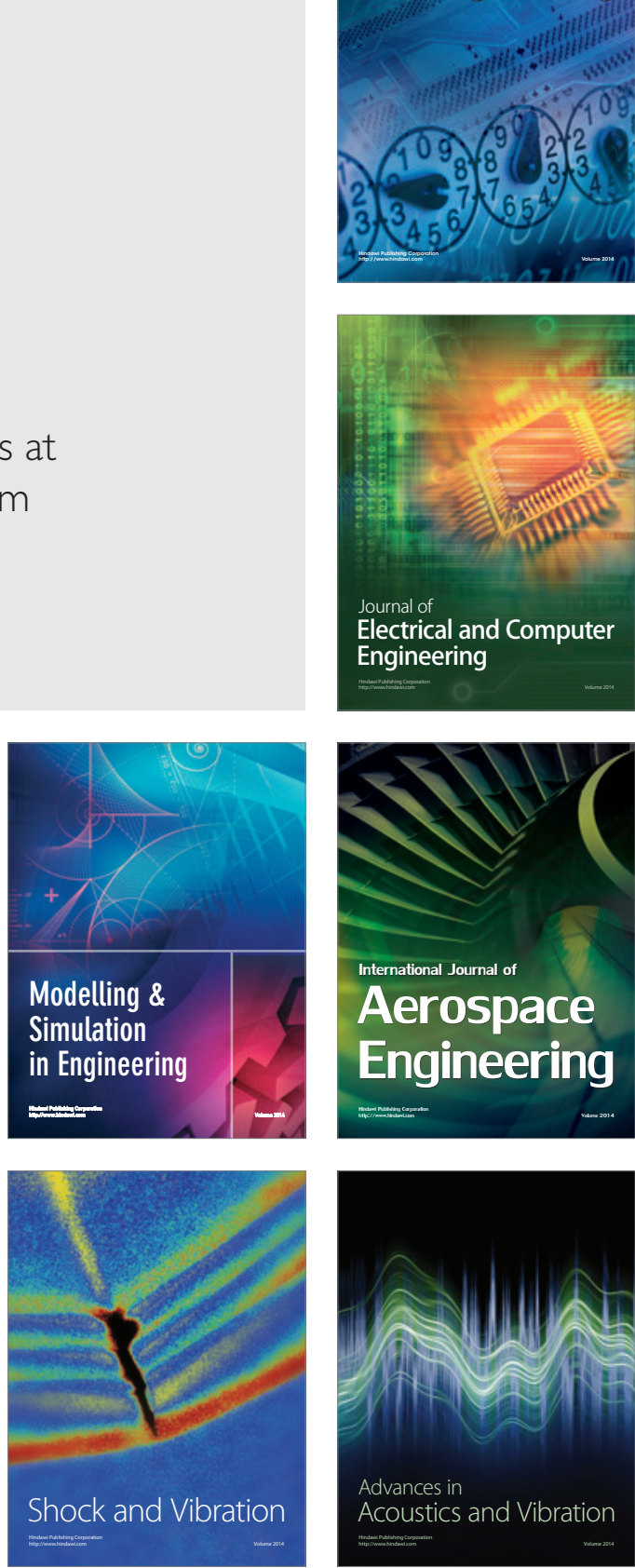